\title{
Influence of Skeletal Muscle Mass and Spinal Alignment on Surgical Outcomes for Lumbar Spinal Stenosis
}

\author{
Yawara Eguchi ${ }^{1}$, Munetaka Suzuki ${ }^{1}$, Hajime Yamanaka ${ }^{1}$, Hiroshi Tamai ${ }^{1}$, Tatsuya Kobayashi ${ }^{1}$, \\ Sumihisa Orita ${ }^{2}$, Kazuyo Yamauchi ${ }^{2}$, Miyako Suzuki ${ }^{2}$, Kazuhide Inage ${ }^{2}$, Kazuki Fujimoto ${ }^{2}$, \\ Hirohito Kanamoto ${ }^{2}$, Koki Abe ${ }^{2}$, Masaki Norimoto ${ }^{2}$, Tomotaka Umimura ${ }^{2}$, Yasuchika Aoki ${ }^{3}$, Masao Koda ${ }^{2}$, \\ Takeo Furuya ${ }^{2}$, Tomoaki Toyone ${ }^{4}$, Tomoyuki Ozawa ${ }^{4}$, Kazuhisa Takahashi ${ }^{2}$, Seiji Ohtori ${ }^{2}$ \\ ${ }^{1}$ Department of Orthopaedic Surgery, Shimoshizu National Hospital, Yotsukaido, Japan \\ ${ }^{2}$ Department of Orthopaedic Surgery, Chiba University Graduate School of Medicine, Chiba, Japan \\ ${ }^{3}$ Department of Orthopaedic Surgery, Eastern Chiba Medical Center, Chiba, Japan \\ ${ }^{4}$ Department of Orthopaedic Surgery, Showa University School of Medicine, Tokyo, Japan
}

Study Design: Retrospective observational study.

Purpose: We considered the relationship between spinal alignment and skeletal muscle mass on clinical outcomes following a surgery for lumbar spinal stenosis (LSS).

Overview of Literature: There are no reports of preoperative factors predicting residual low back pain following surgery for LSS.

Methods: Our target population included 34 women (mean age, 74.4 years) who underwent surgery for LSS. Prior to and 6 months after the surgery, systemic bone mineral density and lean soft tissue mass were measured using dual-energy $\mathrm{X}$-ray absorptiometry. Skeletal muscle mass index (SMI) was calculated as the sum of the arm and leg lean mass in kilograms divided by height in meters squared. The spinal alignment was also measured. Clinical outcomes were evaluated using the Japanese Orthopedic Association scoring system, leg and low back pain Visual Analog Scale, and Roland-Morris Disability Questionnaire (RDQ). Additionally, we examined the bone mineral density, skeletal muscle mass, and spinal alignment before and after the surgery. We used the Spearman correlation coefficient to examine the associations among clinical outcomes, preoperative muscle mass, and spinal alignment. Results: Sarcopenia (SMI <5.46) was observed in nine subjects (26.5\%). Compared with normal subjects (SMI >6.12), RD0 was significantly higher in subjects with sarcopenia $(p=0.04)$. RDO was significantly negatively correlated with $S M I(r=-0.42, p<0.05)$. There was a significant positive correlation between postoperative $\mathrm{RDQ}$ and pelvic tilt (PT; $r=0.41, p<0.05$ ). SMI and PT were significantly negatively correlated $(r=-0.39, r<0.05)$.

Conclusions: Good postoperative outcomes were negatively correlated with low preoperative appendicular muscle mass, suggesting that postoperative outcomes were inferior in cases of decreased appendicular muscle mass (sarcopenia). Posterior PT due to decreased limb muscle mass may contribute to postoperative back pain, showing that preoperatively reduced limb muscle mass and posterior PT are predictive factors in the persistence of postoperative low back pain.

Keywords: Sarcopenia; Skeletal muscle; Spinal stenosis; Low back pain; Surgery

Received Aug 23, 2017; Revised Oct 4, 2017; Accepted Oct 30, 2017

Corresponding author: Yawara Eguchi

Department of Orthopaedic Surgery, Shimoshizu National Hospital, 934-5, Shikawatashi, Yotsukaido, Chiba, 284-0003, Japan

Tel: +81-43-422-2511, Fax:+81-43-421-3007, E-mail: yawara_eguchi@yahoo.co.jp 


\section{Introduction}

An increase in the aging population has led to an increased number of patients with spinal disorders. Intervertebral disc lesions and lumbar nerve root disorders often cause low back pain (LBP). Lumbar spinal stenosis (LSS) is one of many causes of chronic LBP [1]. Although, symptomatic LSS causes spinal claudication, back pain, and radicular leg pain, Jones et al. [2] reported a significant improvement in LBP in patients with LSS who underwent spinal decompression. We previously demonstrated that in addition to leg pain, LBP is also significantly improved by spinal decompressive surgery with appropriate diagnosis in patients with LSS [3].

Nevertheless, some patients with postoperative LBP or lower extremity pain have not experienced operative complications. Loss of lordosis is thought to be one of the factors influencing the postoperative residual symptoms $[4,5]$.

Schwab et al. $[6,7]$ clearly showed that postoperative global sagittal balance is significantly correlated with postoperative residual pain and disability. They showed that radiological parameters such as the sagittal vertical axis (SVA), pelvic tilt (PT), and balance of pelvic incidence (PI) and lumbar lordosis (LL) are mostly correlated with pain and disability. Lafage et al. [8] found an association between posterior PT and stooping posture with poor quality of life (QOL), and considered PT and SVA to be vital factors for pain and disability.

Sarcopenia is a syndrome characterized by progressive and systemic loss of skeletal muscle mass and strength. It is a high risk state wherein a fall could easily lead to a patient becoming bedridden, and it can lead to major physical and economic losses in an aging society [9-11]. Sarcopenia is believed to be caused by inactivity; however, the mechanism has not yet been completely elucidated.

Eguchi et al. [12] previously reported that decreases in pelvic/lumbar support structures, such as trunk and appendicular muscle mass, may be involved in the progression of spinal deformities and an increased LBP. Appendicular skeletal muscle was related to posterior PT. Moreover, LBP was negatively correlated with appendicular skeletal muscle mass and positively correlated with PT, suggesting that sarcopenia may be associated with LBP due to its association with posterior PT [12].

Although it seems likely that reductions in skeletal muscle mass would affect surgical outcomes, the relationship between skeletal muscle mass and surgical outcomes in LSS has not yet been elucidated.

In the present study, we considered the relationship between spinal column alignment and skeletal muscle mass on clinical outcomes following surgery for LSS.

\section{Materials and Methods}

\section{Subjects}

Among the 140 patients who underwent surgery for LSS at our institution between April 2014 and October 2016, 34 women (mean age, 74.4 years) who filled clinical questionnaires before and at least 6 months after surgery were reviewed. There were 17 cases of single-level spinal stenosis, 10 cases of double-level spinal stenosis, and seven cases of foraminal stenosis. Before surgery, foraminal stenosis was comprehensively diagnosed based on clinical symptoms, physical findings, plain X-rays, computed tomography, magnetic resonance imaging (MRI), and three-dimensional (3D)-MRI. Foraminal stenosis was defined based on abnormalities such as nerve indentation, swelling, and running transversely in their course through the foramen on 3D-MRI. The nerve roots were selectively blocked to diagnose damaged nerve roots based on function. If the Visual Analog Scale (VAS, 100-mm method) of the lower limbs was alleviated by $\leq 20 \mathrm{~mm}$ at 30 minutes after nerve root block, the diagnosis was considered positive [3].

There were 11 cases of $\mathrm{L} 3 / 4,28$ cases of $\mathrm{L} 4 / 5$, and seven cases of L5/S1 stenosis. The procedures included 17 cases of single-level laminectomy, 10 cases of double-level laminectomy, five cases of single-level transforaminal lumbar interbody fusion (TLIF), one case of double-level TLIF, and one case of single-level oblique lateral interbody fusion (OLIF) (Table 1). LSS was diagnosed in all patients using laminectomy, and foraminal stenosis was diagnosed in all patients using TLIF or OLIF. The exclusion criteria included a history of multiple fractures of the thoracolumbar spine, spinal or hip joint surgery, and neuromuscular disorders, such as Parkinson's disease.

\section{Measurement of body composition}

Body composition was measured using a whole-body, dual-energy X-ray absorptiometry (DXA, QDR-DELPHIW scanner DPX-NT; Hologic, Waltham, MA, USA), which assessed the mass of lean soft tissue, fat, and bone mineral for the whole body and specific regions such as the arms, 
legs, and trunk. Appendicular skeletal muscle mass was calculated as the sum of skeletal muscle mass in the arms and legs, assuming that the mass of lean soft tissue was representative of the skeletal muscle mass. Skeletal muscle mass index (SMI) was calculated as four-limb lean soft tissue mass in kilograms divided by the height in meters squared. Sarcopenia was defined as (appendicular) SMI $<5.46 \mathrm{~kg} / \mathrm{m}^{2}$, based on normalized data for sarcopenia in Japanese men and women [13]. Trunk SMI was defined as the trunk lean mass in kilograms divided by height in meters squared, although the lean mass of the trunk also includes the internal organs. The whole-body bone mineral density (BMD), arm lean mass, leg lean mass, trunk lean mass, appendicular lean mass, and total lean mass values from before and 6 months after the surgery were recorded for all patients (Table 1).

\section{Radiographic measurements}

The frontal view of the entire spine and the lateral view including the hip joints were photographed with the sub-

Table 1. Patient characteristics

\begin{tabular}{lc}
\hline Characteristic & Value \\
\hline Age (yr) & $74.4 \pm 7.6$ \\
\hline Height (m) & $1.48 \pm 0.06$ \\
\hline Weight $(\mathrm{kg})$ & $50.8 \pm 8.2$ \\
\hline Body mass index $\left(\mathrm{kg} / \mathrm{m}^{2}\right)$ & $23.3 \pm 3.3$ \\
\hline Diagnosis & \\
\hline Single-level stenosis & 17 \\
\hline Double-level stenosis & 10 \\
\hline Foraminal stenosis & 7 \\
\hline Level of stenosis & 11 \\
\hline L3/4 & 28 \\
\hline L4/5 & 7 \\
\hline L5/S1 & 17 \\
\hline Surgery & 10 \\
\hline Laminectomy & \\
\hline One level & 1 \\
\hline Two levels & \\
\hline Transforaminal lumbar interbody fusion & \\
\hline One level & \\
\hline Two levels & \\
\hline Oblique lateral interbody fusion (one level) & \\
\hline
\end{tabular}

Values are presented as mean \pm standard deviation or number. jects in a standing position and conducted before and after the surgery for three cases of fusion and 19 cases of decompression. Radiographic measurements comprised lumbar scoliosis (LS), SVA, thoracic kyphosis (TK), LL, PT, PI, sacral slope (SS), and PI-LL. LS was measured as the angle between the lower end plates of L1 and L5 on the frontal radiographs. SVA was measured as the distance from the $\mathrm{C} 7$ plumb line to a perpendicular line drawn from the superior posterior end plate of the S1 vertebral body on the lateral radiographs. TK was measured from the upper end plate of T5 to the lower end plate of T12. LL was measured from the lower end plate of T12 to the upper end plate of S1. PT was measured as the angle between the vertical line and the line joining the hip axis to the center of the superior end plate of S1. PI was measured as the angle subtended by a perpendicular line from the upper end plate of $\mathrm{S} 1$ and a line connecting the center of the femoral head to the center of the cephalad end plate of S1. Finally, SS was measured as the angle between the superior end plate of $\mathrm{S} 1$ and a horizontal line.

\section{Measurement of clinical outcomes}

Before and 6 months after the surgery, clinical outcomes were evaluated using a VAS score for LBP and leg pain ranging from 0 (no pain) to 100 (extreme amount of pain), the Japanese Orthopedic Association (JOA) scoring system (range, 0-29 points), and the Roland-Morris Disability Questionnaire (RDQ; range, 0-24 points). The normal JOA score is 29 points, based on three subjective symptoms ( 9 points), three clinical signs including straight-leg raise (6 points), and seven activities of daily living (ADL, 14 points). The normal RDQ score is 0 point, and the total number of items checked ranges from zero to 24 .

Sarcopenia prevalence in each group, correlations between appendicular and trunk SMIs and spinal alignment with clinical and surgical outcomes, and correlation between spinal alignment and appendicular and trunk SMIs were studied.

\section{Statistical analysis}

Statistical analyses were performed using Stat View software ver. 5.0 (SAS Institute Inc., Cary, NC, USA). For each parameter, difference between both the groups was evaluated using an unpaired $t$-test. Pearson correlation 
coefficients were calculated to determine the correlation between appendicular SMI or trunk SMI and spinal parameters or clinical outcomes. A threshold of $p<0.05$ was considered statistically significant.

\section{Ethics and consent to participate}

All protocols involving humans were approved by the Shimoshizu National Hospital (IRB approval no., H26'6) and and were performed in accordance with the ethical standards laid down in the 1964 Declaration of Helsinki and its later amendments. All participants provided a written informed consent prior to their inclusion in this study.

\section{Results}

The preoperative radiographical alignment measures included LS $\left(8.5^{\circ} \pm 10.4^{\circ}\right)$, SVA $(46.2 \pm 30.5 \mathrm{~mm})$, TK $\left(22.8^{\circ} \pm 9.7^{\circ}\right)$, LL $\left(38.2^{\circ} \pm 14.5^{\circ}\right)$, PI $\left(49.2^{\circ} \pm 10.9^{\circ}\right)$, PT $\left(25.1^{\circ} \pm 8.2^{\circ}\right)$, SS $\left(27.6^{\circ} \pm 9.9^{\circ}\right)$, and PI-LL $\left(11.0^{\circ} \pm 13.5^{\circ}\right)$. The postoperative radiographical alignment measures included LS $\left(7.9^{\circ} \pm 8.4^{\circ}, p=0.82\right)$, SVA $(37.6 \pm 24.3 \mathrm{~mm}$, $p=0.28)$, TK $\left(21.0^{\circ} \pm 8.7^{\circ}, p=0.47\right)$, LL $\left(39.4^{\circ} \pm 13.9^{\circ}\right.$, $p=0.75)$, PI $\left(51.4^{\circ} \pm 12.3^{\circ}, p=0.75\right)$, PT $\left(24.5^{\circ} \pm 9.0^{\circ}, p=0.8\right)$, SS $\left(29.0^{\circ} \pm 10.1^{\circ}, p=0.62\right)$, and PI-LL $\left(12.0^{\circ} \pm 13.0^{\circ}, p=0.79\right)$. There were no significant changes in these parameters before and after the surgery (Table 2).

Sarcopenia (SMI <5.46) was observed in nine out of the 34 subjects $(26.5 \%)$; presarcopenia (SMI $<6.12)$ was observed in 12 out of the 34 subjects (35.3\%). RDQ scores in sarcopenia (SMI <5.46), presarcopenia (SMI <6.12), and normal subjects (SMI >6.12) were 9.67 $\pm 7.47,6.00 \pm 4.63$, and $4.38 \pm 3.88$, respectively. Compared with normal subjects, RDQ was significantly higher in subjects with sarcopenia $(p=0.04)$; however, these differences were not significant among subjects with presarcopenia $(p=0.34)$ (Fig. 1).

BMD before $\left(0.96 \pm 0.09 \mathrm{~g} / \mathrm{cm}^{2}\right)$ and after $(0.97 \pm 0.09 \mathrm{~g} /$ $\left.\mathrm{cm}^{2}\right)$ the surgery was not significantly different $(p=0.65)$. Appendicular SMI was $5.96 \pm 0.72$ before and $5.76 \pm 0.65$ after the surgery, and although a decreasing trend was observed, there was no significant difference $(p=0.25)$. Trunk SMI was $7.48 \pm 0.76$ before and $7.67 \pm 0.81$ after the surgery, and while an increasing trend observed, there was no significant difference $(p=0.34)$.

Table 2. Comparisons of pre- and postoperative measurements of spinal alignment, BMD, skeletal muscle mass, and clinical outcome

\begin{tabular}{|c|c|c|c|}
\hline Variable & Preoperative & Postoperative & $p$-value \\
\hline \multicolumn{4}{|l|}{ Spinal alignment } \\
\hline Lumbar scoliosis $\left({ }^{\circ}\right)$ & $8.5 \pm 10.4$ & $7.9 \pm 8.4$ & 0.82 \\
\hline Sagittal vertical axis (mm) & $46.2 \pm 30.5$ & $37.6 \pm 24.3$ & 0.28 \\
\hline Thoracic kyphosis $\left({ }^{\circ}\right)$ & $22.8 \pm 9.7$ & $21.0 \pm 8.7$ & 0.47 \\
\hline $\operatorname{LL}\left({ }^{\circ}\right)$ & $38.2 \pm 14.5$ & $39.4 \pm 13.9$ & 0.75 \\
\hline $\mathrm{PI}\left({ }^{\circ}\right)$ & $49.2 \pm 10.9$ & $51.4 \pm 12.3$ & 0.75 \\
\hline Pelvic tilt $\left({ }^{\circ}\right)$ & $25.1 \pm 8.2$ & $24.5 \pm 9.0$ & 0.8 \\
\hline Sacral slope $\left({ }^{\circ}\right)$ & $27.6 \pm 9.9$ & $29.0 \pm 10.1$ & 0.62 \\
\hline $\mathrm{PI}-\mathrm{LL}\left({ }^{\circ}\right)$ & $11.0 \pm 13.5$ & $12.0 \pm 13.0$ & 0.79 \\
\hline $\mathrm{BMD}\left(\mathrm{g} / \mathrm{cm}^{2}\right)$ & $0.96 \pm 0.09$ & $0.97 \pm 0.09$ & 0.65 \\
\hline \multicolumn{4}{|l|}{ Skeletal muscle mass } \\
\hline SMI & $5.96 \pm 0.72$ & $5.76 \pm 0.65$ & 0.25 \\
\hline Trunk SMI & $7.48 \pm 0.76$ & $7.67 \pm 0.81$ & 0.34 \\
\hline \multicolumn{4}{|l|}{ Clinical outcome } \\
\hline Japanese Orthopedic Association score & $17.2 \pm 3.4$ & $23.5 \pm 3.4$ & $9.2 \times 10^{-11}$ \\
\hline Roland-Morris Disability Questionnaire & $10.5 \pm 4.9$ & $6.4 \pm 5.6$ & 0.002 \\
\hline Visual Analog Scale (low back pain) & $53.2 \pm 26.5$ & $28.8 \pm 26.2$ & 0.0004 \\
\hline
\end{tabular}

Values are presented as mean \pm standard deviation.

BMD, bone mineral density; LL, lumbar lordosis; PI, pelvic incidence; SMI, skeletal muscle mass index. 


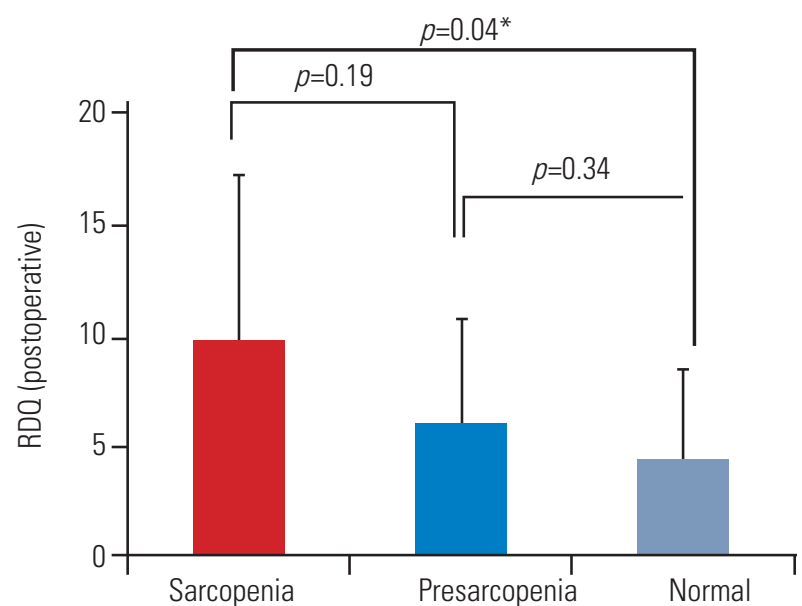

Fig. 1. Postoperative RDQ in sarcopenia, presarcopenia, and normal

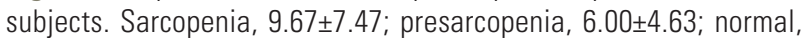
$4.38 \pm 3.88$. There was a significant increase in postoperative RD0 among subjects with sarcopenia $(p=0.04)$, but these differences were not significant among the subjects with presarcopenia $(p=0.34)$. RD0, Roland-Morris Disability Questionnaire. ${ }^{*} p<0.05$.

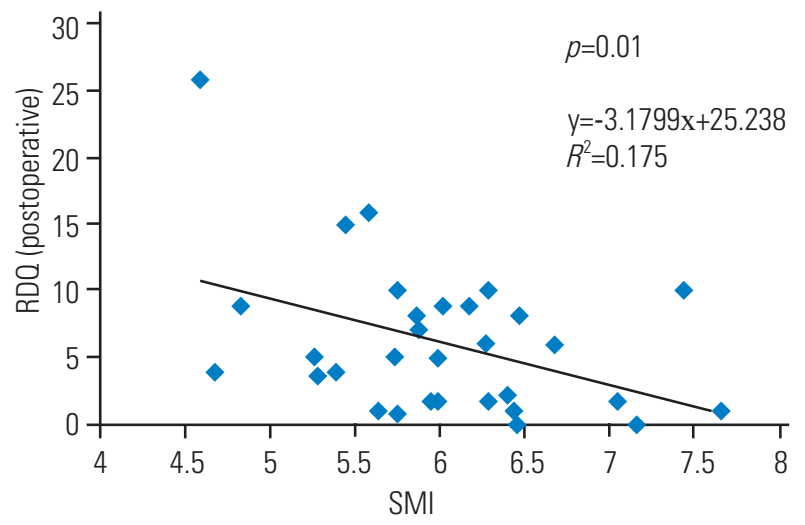

Fig. 2. Correlations between appendicular and trunk SMls and clinical symptoms. A statistically significant negative correlation was noted between appendicular SMI and postoperative RDO $(p<0.05)$. SMI, skeletal muscle mass index; RDQ, Roland-Morris Disability Questionnaire.

The clinical outcomes included a JOA score of $17.2 \pm 3.4$ before and $23.5 \pm 3.4$ after the surgery, a RDQ of $10.5 \pm 4.9$ before and 6.4 \pm 5.6 after the surgery, and the VAS score for LBP was $53.2 \pm 26.5$ before and $28.8 \pm 26.2$ after the surgery; LBP significantly improved $(p<0.05)$ (Table 2). There were no complications during and after the surgeries.

Regarding correlation between clinical symptoms and surgical outcomes with skeletal muscle mass, the postoperative RDQ showed a significant negative correlation with SMI $(r=-0.42, p<0.05)$ (Fig. 2). There were no correlations between other clinical outcomes such as VAS scores, JOA scores, and skeletal muscle mass. Clinical
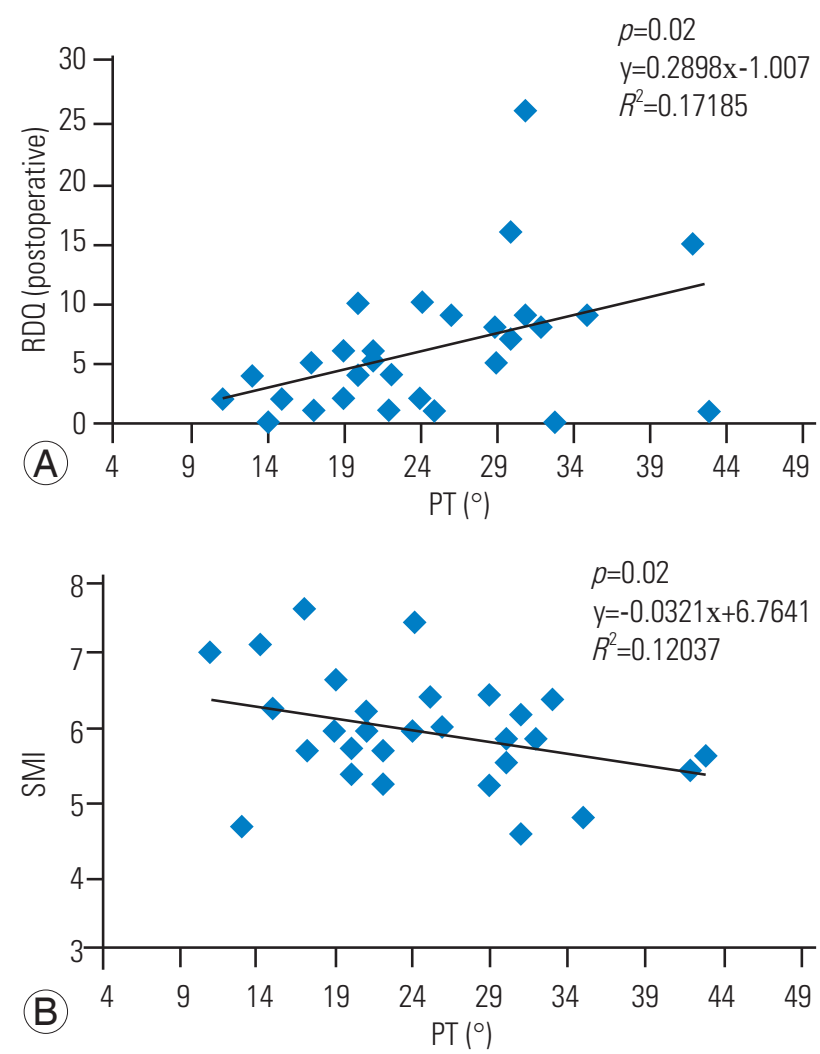

Fig. 3. Correlation between spinal alignment and appendicular and trunk SMls. (A) A statistically significant positive correlation was noted between PT and postoperative RD0 ( $p<0.05)$. (B) A statistically significant negative correlation was noted between PT and appendicular SMI ( $p<0.05)$. SMI, skeletal muscle mass index; PT, pelvic tilt; RDQ, Roland-Morris Disability Questionnaire.

outcomes and spinal column alignment showed a significant positive correlation between postoperative RDQ and PT $(r=0.41, p<0.05)$ (Fig. 3A). Correlations between spinal column alignment and skeletal muscle mass showed that SMI and PT were significantly negatively correlated $(r=-0.39, r<0.05)$ (Fig. 3B).

\section{Discussion}

Sarcopenia is defined as an age-associated loss of skeletal muscle mass and function with a risk of adverse outcomes such as physical disability and poor QOL $[9,10]$. Sarcopenia is very common in older individuals, with a reported prevalence of 5\%-13\% in 60-70-year-olds [11].

In a report on sarcopenia and spinal diseases, Miyakoshi et al. [14] noted that $20 \%$ of Japanese patients with osteoporosis suffer from sarcopenia complications whereas only $10 \%$ of healthy individuals have sarcopenia. Eguchi et al. [12] previously reported a high sarcopenia preva- 
lence rate of $46 \%$ in patients with degenerative LS and $70 \%$ in those with dropped head syndrome [15]. In the present study, sarcopenia was observed in $26.5 \%$ of LSS surgical subjects. This is somewhat high compared with the reported $10 \%$ prevalence in healthy subjects.

With regard to spinal alignment that adversely affects QOL, Takemitsu et al. [16] reported that $95 \%$ of patients with lumbar degenerative kyphosis suffer from LBP, which severely disrupts their ADL. Glassman et al. [17] found that patients with large SVA, where the C7 plumb line shows anterior displacement, suffer from the greatest disruption of QOL, stressing the importance of sagittal alignment. Lafage et al. [8] associated posterior PT and stooping posture with poor QOL, and considered PT and SVA to be vital factors. Schwab et al. [6,7] demonstrated that SVA, PT, and PI-LL were most closely related to LBP and disability; this group chose radiographical parameters PI-LL $<10^{\circ}, \mathrm{PT}<20^{\circ}$, and SVA $<50 \mathrm{~mm}$ as the thresholds for correction. Aoki et al. [18] revealed that postoperative PI-LL mismatch caused greater residual LBP after shortsegment lumbar interbody fusion.

Nevertheless, PI-LL was used to evaluate alignment following surgery. There are currently no reports of preoperative factors predicting residual LBP. In the present study, good postoperative LSS results were negatively correlated with low preoperative appendicular muscle mass. Specifically, the postoperative outcomes were inferior in cases of decreased appendicular muscle mass (sarcopenia).

There were no significant differences between the various spinal alignment parameters before and after surgery. Furthermore, no correlation was found between SVA, PILL, and postoperative LBP. Further investigation of alignment change was not required in 22 cases.

Eguchi et al. [12] reported that the appendicular skeletal muscle was related to posterior PT; moreover, RDQ was negatively correlated with appendicular skeletal muscle mass and positively correlated with PT, suggesting that sarcopenia may be associated with LBP as a result of posterior PT. In the present study, appendicular muscle mass reduction and posterior PT were related to LBP. It is possible that posterior PT caused by the appendicular muscle mass reduction contributed to postoperative LBP. To the best of our knowledge, this is the first report to show that preoperatively reduced SMI and posterior PT are predictive factors for the persistence of the postoperative lower back pain.

Our study has several limitations. Firstly, the number of subjects included was small and they had short postoperative follow-up periods; therefore, our findings need to be confirmed in a larger population with longer postoperative follow-up periods. Secondly, our study population included only Japanese women with low body mass indices. Thirdly, DXA cannot measure individual spinal muscles such as the paravertebral and psoas muscles. The trunk SMI defined in this study included internal organs; therefore, it is not an accurate measure of actual trunk muscle volume but merely a relative evaluation. Lastly, postoperative residual symptoms are affected by multiple factors.

In this study, 27 cases of decompression and seven cases of fusion surgery were included. Furthermore, back pain is affected by differences in surgical procedures and ensuing spinal alignment changes.

In cases of decompression, spinal alignment measurements (preoperative, postoperative, $p$-value) included LS (6.9, 6.6, $p=0.92)$, SVA $(46.3,39.4, p=0.49)$, TK $(23.8,21.5$, $p=0.38)$, LL (41.0, 41.7, $p=0.87)$, PI (50.0, 54.7, $p=0.21$ ), PT (24.4, 26.2, $p=0.52)$, SS $(29.6,30.2, p=0.85)$, and PI-LL $(9.0,13.1, p=0.34)$. There were no significant changes in alignment between before and after surgery.

In cases of fusion, spinal alignment measurements (preoperative, postoperative, $p$-value) included LS (14.4, 10.7, $p=0.60)$, SVA $(46.8,33.7, p=0.31)$, TK $(19.1,19.7, p=0.93)$, LL (28.0, 34.6, $p=0.47)$, PI $(46.3,44.3, p=0.73)$, PT (27.7, $20.9, p=0.12)$, SS $(20.7,26.4, p=0.23)$, and PI-LL $(18.3,9.7$, $p=0.26$ ). Slight improvements were observed in PT and PI-LL mismatch, but otherwise there were no significant changes in alignment between before and after surgery. Further investigation is required with a large number of cases.

\section{Conclusions}

We considered the relationship between spinal alignment and skeletal muscle mass on clinical outcomes following surgery for LSS. Sarcopenia was found in $26.5 \%$ of cases of LSS. Good postoperative outcomes were negatively correlated with low preoperative appendicular muscle mass, suggesting that postoperative outcomes were inferior in cases of decreased appendicular muscle mass (sarcopenia). We also found that reduced appendicular muscle mass was associated with posterior PT and LBP. Posterior PT due to appendicular muscle mass reduction may contribute to postoperative LBP. A reduced preoperative appendicular muscle mass and posterior PT may be predictive 
factors for postoperative residual LBP.

\section{Conflict of Interest}

No potential conflict of interest relevant to this article was reported.

\section{References}

1. Ganz JC. Lumbar spinal stenosis: postoperative results in terms of preoperative posture-related pain. J Neurosurg 1990;72:71-4.

2. Jones $\mathrm{AD}$, Wafai $\mathrm{AM}$, Easterbrook AL. Improvement in low back pain following spinal decompression: observational study of 119 patients. Eur Spine J 2014;23:135-41.

3. Eguchi Y, Suzuki M, Yamanaka H, et al. Assessment of clinical symptoms in lumbar foraminal stenosis using the Japanese Orthopaedic Association Back Pain Evaluation Questionnaire. Korean J Spine 2017;14:16.

4. La Grone MO. Loss of lumbar lordosis: a complication of spinal fusion for scoliosis. Orthop Clin North Am 1988;19:383-93.

5. Kostuik JP, Maurais GR, Richardson WJ, Okajima Y. Combined single stage anterior and posterior osteotomy for correction of iatrogenic lumbar kyphosis. Spine (Phila Pa 1976) 1988;13:257-66.

6. Schwab FJ, Blondel B, Bess S, et al. Radiographical spinopelvic parameters and disability in the setting of adult spinal deformity: a prospective multicenter analysis. Spine (Phila Pa 1976) 2013;38:E803-12.

7. Schwab F, Ungar B, Blondel B, et al. Scoliosis Research Society-Schwab adult spinal deformity classification: a validation study. Spine (Phila Pa 1976) 2012;37:1077-82.

8. Lafage V, Schwab F, Patel A, Hawkinson N, Farcy JP. Pelvic tilt and truncal inclination: two key radiographic parameters in the setting of adults with spinal deformity. Spine (Phila Pa 1976) 2009;34:E599606.
9. Cruz-Jentoft AJ, Landi F, Schneider SM, et al. Prevalence of and interventions for sarcopenia in ageing adults: a systematic review: report of the International Sarcopenia Initiative (EWGSOP and IWGS). Age Ageing 2014;43:748-59.

10. Wu IC, Lin CC, Hsiung CA, et al. Epidemiology of sarcopenia among community-dwelling older adults in Taiwan: a pooled analysis for a broader adoption of sarcopenia assessments. Geriatr Gerontol Int 2014;14 Suppl 1:52-60.

11. Morley JE. Sarcopenia: diagnosis and treatment. J Nutr Health Aging 2008;12:452-6.

12. Eguchi Y, Suzuki M, Yamanaka H, et al. Associations between sarcopenia and degenerative lumbar scoliosis in older women. Scoliosis Spinal Disord 2017;12:9.

13. Sanada K, Miyachi M, Tanimoto M, et al. A crosssectional study of sarcopenia in Japanese men and women: reference values and association with cardiovascular risk factors. Eur J Appl Physiol 2010;110:5765.

14. Miyakoshi N, Hongo M, Mizutani Y, Shimada Y. Prevalence of sarcopenia in Japanese women with osteopenia and osteoporosis. J Bone Miner Metab 2013;31:556-61.

15. Eguchi Y, Toyoguchi T, Koda M, et al. The influence of sarcopenia in dropped head syndrome in older women. Scoliosis Spinal Disord 2017;12:5.

16. Takemitsu Y, Harada Y, Iwahara T, Miyamoto M, Miyatake Y. Lumbar degenerative kyphosis: clinical, radiological and epidemiological studies. Spine (Phila Pa 1976) 1988;13:1317-26.

17. Glassman SD, Bridwell K, Dimar JR, Horton W, Berven S, Schwab F. The impact of positive sagittal balance in adult spinal deformity. Spine (Phila Pa 1976) 2005;30:2024-9.

18. Aoki Y, Nakajima A, Takahashi H, et al. Influence of pelvic incidence-lumbar lordosis mismatch on surgical outcomes of short-segment transforaminal lumbar interbody fusion. BMC Musculoskelet Disord 2015;16:213. 